\title{
ABUNDANCE STRATIFICATIONS IN THE ATMOSPHERES OF Ap STARS: THE CASE OF GALLIUM
}

\author{
G. Alecian and M.-C. Artru \\ Observatoire de Paris-Meudon \\ F-92195 Meudon Principal Cedex \\ France
}

\begin{abstract}
The abundances are usually determined assuming homogeneous concentrations for all elements in the line forming region. If diffusion driven by radiation occurs, this condition is not verified and the element stratification affects the equivalent widths as well as the line profiles. We shall illustrate these problems by a study of the gallium case. A synthetic spectrum of the Ga II resonance line $\lambda 1414.401 \AA$ has been computed assuming that gallium is stratified according a recent theoretical study of the diffusion of this element.
\end{abstract}

\section{INTRODUCTION}

The Ap stars are known to have strong abundance anomalies: overabundances may be, in some cases up to $10^{5}$ for metals and sometimes underabundances are detected (for instance for helium). These anomalies are explained by invoking the diffusion process: elements migrate through the outer stellar layers pushed up by the radiation field. This process is more or less efficient according to the element. Many papers have appeared on the subject and one may refer, as a first reading, to the basic paper of Michaud (1970) and also to the review by Bonsack \& Wolff (1980) and the one by Alecian \& Vauclair (1983).

The diffusion process is fundamentally time-dependent. In about $10^{4}-10^{5}$ years, it leads to the appearance of abundance stratifications in the Ap stars' atmospheres. In this case, an element may be strongly enhanced in some places and deficient in an other places. According to where the spectral lines of this precise element are formed, it may be detected over-, under-abundant or... normal. Of course, this description is very schematized, the process is much more complex since other effects like magnetic fields, macroscopic motions of the whole matter may interact with diffusion.

Apart the abundance anomalies which are generally inferred from the equivalent widths, the stratification may change (for stratified elements) the spectral line profiles (see for instance, the Ca study by Borsenberger et al, 1981), and also curves of growth (Alecian, 1982). In the present paper we shall consider the effects of stratification, on the $\mathrm{Ga} I I$ resonance line $\lambda 1414.401 \AA$.

\section{THE GALLIUM STRATIFICATION}

The gallium abundances have been recently determined in a relatively large sample of Ap stars by Takada-Hidai et al (1986) on the basis of IUE observations of the UV resonance 
lines of Ga II and III. These data complete and improve previous determinations by Heacox (1979) and Guthrie (1984). They confirm that the largest gallium overabundances occur in $\mathrm{Hg}-\mathrm{Mn}$ stars and that they are generally smaller in Ap-Si stars. A comparison of these stars, using the photometric index $\delta_{1400}$ of Jamar et al (1978) which measure the well known flux depression at $1400 \AA$ (identified as to be related to silicon overabundance by Artru, 1986), shows that the overabundance of gallium may be anti-correlated with the overabundance of silicon.

\subsection{Recent results on the gallium diffusion}

A recent theoretical study of the gallium diffusion motivated by these new data, have been made by Alecian \& Artru (1987) using up-to-date gallium atomic data. They concluded that, if mass loss effects are negligible, the gallium overabundances should appear weaker in the presence of horizontal magnetic field. Schematically, in Ap-Si stars one might expect that patches of gallium are located where magnetic field is vertical while patches of silicon are located at places where magnetic field is horizontal. On the other hand, they have shown that the upper limit of the observed gallium overabundances are well explained in the framework of the diffusion theory (see their Fig.6).

\subsection{The effect of stratification on the $\mathrm{Ga}$ II resonance line $\lambda 1414.401 \AA$}

In order to test further the diffusion model concerning gallium, we tried to establish more precisely the effect a stratification of gallium on its spectral lines. We chose to study the $\lambda$ 1414.401 A resonance line of Ga II in an atmosphere like those of the most typical $\mathrm{Hg}-\mathrm{Mn}$ star of the sample studied by Takada-Hidai et al (1986): HD 175640 ( $\mathrm{T}_{\text {eff }} \approx 12000 \mathrm{~K}, \log$ $g \approx 3.9$, v.sin $\mathrm{i}<5 \mathrm{~km} . \mathrm{s}^{-1}$ ). The equivalent width measured by these authors for the $\lambda$ $1414.401 \AA$ line is about $1.56 \AA$. Taking this equivalent width, the standard abundance determination (assuming homogeneous gallium) gives that gallium is overabundant by a factor of 3000 with respect to the solar value.

We computed a synthetic spectrum of the $\lambda 1414.401 \AA$ line, using the NLTE model of atmosphere of Borsenberger \& Gros $(1978)\left(T_{\text {eff }}=12000 \mathrm{~K}, \log g=4\right)$. The line was computed in LTE with inhomogeneous abundance of gallium throughout the atmosphere.

Firstly, we determined what kind of stratification of gallium may be expected according to the radiation forces published by Alecian \& Artru (1987). Actually the building of stratification is a time-dependent process which must be computed numerically (Alecian \& Grappin, 1984). However, one may simply assume that equilibrium (zero diffusion velocity everywhere) is reached at the end of this process. This leads to the stratification of gallium shown in Fig. 1 where a cloud-like accumulation occurs around $\tau 5000=1$. Using this stratification deduced a priori from the diffusion model, we synthesized the absorption of the $\lambda 1414.401 \AA$ line of Ga II.

\subsubsection{The equivalent width}

The computation of the synthetic spectrum gives an equivalent width of $1.58 \AA$ which is very close to the observed one $1.56 \AA$. This is explained by the fact that the overabundance of $G a$ in the cloud-like accumulation shown in Fig.1, is not far from a factor of 3000 near the depth $\tau 5000=1$ where the line forms.

Other results obtained with various shape and positions of the cloud, have shown that the equivalent width of this line is very sensitive to the position of the higher edge of the 
cloud: to rise up this cloud would increase strongly the equivalent width since the maximum overabundance in it is stronger than $1.210^{4}$ times the solar abundance.

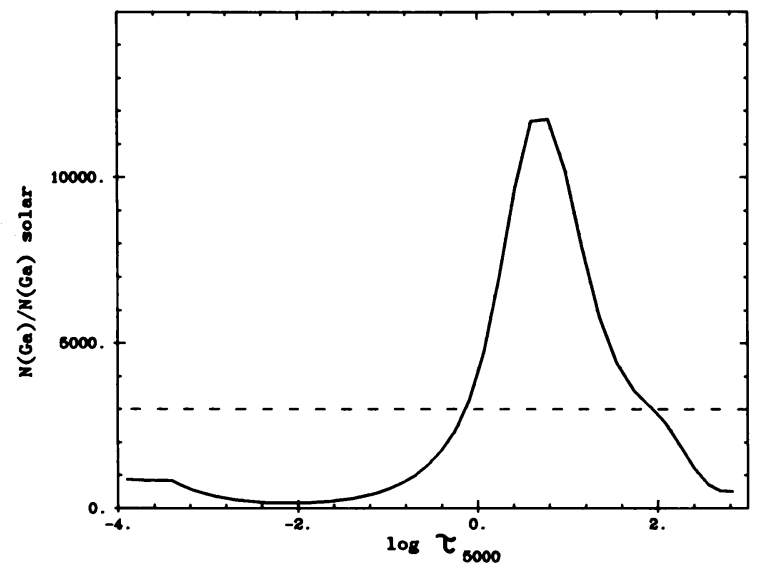

Figure 1. The cloud-like accumulation of gallium (continuous line) deduced from diffusion calculation for $T_{\text {eff }}=12000 \mathrm{~K}, \log g=4$. The plot shows the overabundance of gallium (with respect to solar) vs the logarithm of the optical depth at $5000 \AA$. The dashed line is the overabundance deduced from the standard homogeneous analysis.

\subsubsection{The line profile}

The abundance stratification also affects the line profile. In Fig.2. We show the computed profile of the Ga II resonance line $\lambda 1414.401 \AA$ assuming the same stratification than before.

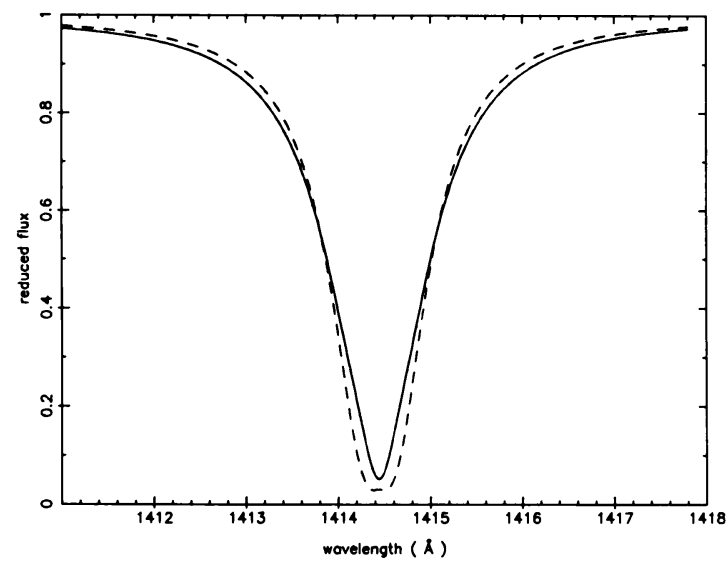

Figure 2. The line profile obtained through a synthetic spectra calculation. The continuous line corresponds to the stratified case (see Fig.1), the dashed line to the homogeneous case.

We compare it to the profile obtained without stratification (dashed line). In this case we have considered an homogeneous overabundance of $x 4200$ with respect to solar, in order to have exactly the same equivalent width $(1.58 \AA)$ in both cases (it is of course slightly 
higher than the measured value). The profiles have been broaden at $0.15 \AA$ which corresponds to a rotational velocity of $5 \mathrm{~km} . \mathrm{s}^{-1}$.

\section{CONCLUSION}

In the present work we have considered the case of a typical $\mathrm{Hg}-\mathrm{Mn}$ star with good observational data and we have studied a gallium line for which the atomic data are reliable. This study has allowed to test a parameter-free model of diffusion: we emphasize that the cloud like accumulation of Fig.1 has been obtained through an approximate diffusion calculation but without any free parameter.

The equivalent width calculated by assuming the cloud-like accumulation of gallium is very close to the observed value.

Concerning the line profile shown in Fig.2, the difference between the stratified and homogeneous cases cannot be detected with IUE spectra, and needs higher resolution and better $\mathrm{S} / \mathrm{N}$. One cannot exclude other ions for which it might be more easy to put into evidence such an effect with present technics.

\section{REFERENCES}

Alecian, G.: 1982, Astron.Astrophys. 107, 61

Alecian, G. and Artru, M.-C.: 1987, Astron. Astrophys., in press

Alecian, G., Grappin, R.: 1984, Astron.Astrophys. 140, 159

Alecian, G., Vauclair, S.: 1983, Fundamentals of Cosmic Physics, 8, 369, Gordon and Breach Science Publishers Ltd.

Artru, M.-C.: 1986, Astron. Astrophys., 168, L5

Bonsack, W.K., Wolff, S.C.: 1980, Astron. J., 85, 599

Borsenberger, J., Gros, M.: 1978, Astron.Astrophys.Suppl. 31, 291

Borsenberger, J., Michaud, G., Praderie, F.: 1981, Astrophys. J., 243, 533

Guthrie, B.N.G.: 1984, Mon. Not. R. Astr. Soc. 206, 85

Heacox, W. D.: 1979, Astrophys. J. 41, 675

Jamar, C., Macau-Hercot, D. and Praderie, F.: 1978, Astron. Astrophys. 63, 155

Michaud,G.: 1970, Astrophys. J. 160, 641

Takada-Hidai, M., Sadakane, K. and Jugaku, J.: 1986, Astrophys. J., 304, 425

\section{DISCUSSION}

JUGAKU: Have you made a similar line profile computation for the Ga III (and possibly $\mathrm{Ga}$ I) resonance line?

ALECIAN: We have only considered two Ga II lines: the UV resonance line at $1414.401 \AA$ and also the visible line 4255.722-.92. Actually the best atomic data and the best observational data are for the UV resonance line.

MARCY: What values of gf, collisional broadening did you use? And what atmosphere did you use?

ALECIAN: The gf of the UV resonance line is 1.8 (it is known within $10 \%$ from recent determination). The collisional broadening is estimated from a semi-classical calculation and may be neglected for the UV line.

We have used the NLTE model atmosphere computed by Borsenberger and Gros (1978). Our synthetic spectrum does not take into account the NLTE effects on the gallium line formation, however we have verified that this approximation is justified. 